\title{
Spun bonding technology and fabric properties: a review
}

\begin{abstract}
The process of making spun bond fabrics combines the production of fabrics with the production of filaments. High process efficiencies and excellent properties of these fabrics have made them acceptable in different areas of application like civil engineering, medical and hygiene, automotive industry, shoe industry and packaging. Controlling the structure and properties of the fabric is a difficult task as there are several process parameters affecting fabric properties besides the structure and properties of the filaments. Several scattered studies explain the influence of process parameters on filament and fabric properties but lot of information on the fabric properties is missing. A comprehensive review of the spun bonding technique, fabric characteristics and the process parameters is presented in this paper. An understanding on this will help in optimizing the process parameters for producing desired properties in the fabric.
\end{abstract}

Keywords: extrusion, polyester, polyurethanes, textiles, thermo-gravimetric analysis, geotextiles, civil engineering, fiber spinning, economics, spin pack filter, plant flexibility, spinning block, electrical method, electrostatic charge, chemical binder methods
Volume I Issue 4 - 2017

Vinay Kumar Midha,' Arjun Dakuri²

'Department of Textile Technology, Dr B R Ambedkar National Institute of Technology, India

${ }^{2}$ Department of Textile Technology,Vignan's University, India

Correspondence: DrVinay Kumar Midha, Department of Textile Technology, Dr B R Ambedkar National Institute of Technology Jalandhar, India- I440I I, India, Emailmidhav@rediffmail.com

Received:September 28, 2016 | Published: April 17, 2017

\section{Introduction}

Spun bond nonwoven fabrics are composed of continuous filaments produced by an integrated fiber spinning, web formation and bonding process. As it eliminates intermediate steps, it is the shortest textile route from polymer to fabrics in one stage and provides opportunities for increasing production and reduction of cost. ${ }^{1}$ Although the technology was developed simultaneously in Europe and United States in the late 1950s but its potential for commercial applications was recognized only in 1960s. In the recent past spun bond nonwovens have developed rapidly due to their excellent properties and high process efficiency. Presently it occupies the largest share among the various techniques of nonwoven fabric manufacture and finds application in different fields like diapers and incontinence products, automotives, geotextiles, civil engineering, medical and packaging.

\section{Production process}

The production process involves four simultaneous and integrated operations viz. filament spinning, drawing, lay down and bonding. The first three operations are directly adopted from conventional manmade fiber spinning. Although any of the spinning techniques, viz. melt, dry and wet can be used but melt spinning is most widely used because of its simplicity and economics. The main function of this system is to solidify and draw the extruded filaments from the spinneret and deposit them onto a conveyor belt. The last operation is web consolidation, whereby strength is provided to the web through mechanical, chemical or thermal bonding methods. ${ }^{2,3}$ Figure 1 show the various steps involved in the spun bonding process.

The key components of the spun bonding line are extruder, filter, metering pump, spinning block, quenching, drawing, web forming, bonding and winding Figure 2 . The extruder consists of a barrel, screw and the channel as shown in Figure 3. Screw helps to guide the molten melt homogeneously into the gear pump through filters. Different kinds of screws are available, but single screw is commonly used.
Twin screw extruder shows excellent mixing capabilities for polymer additives and offer plant flexibility. The amount of polymer in the barrel is very small and hence it is easy to change the line from one polymer to another. Kobelco spun bond plant is capable of producing polypropylene and polyester spun bond in one line. The operation spinning speed is $4700-5000 \mathrm{~m} / \mathrm{min}$ for polyester and $3400-3700 \mathrm{~m} /$ min for polypropylene..$^{4-6}$

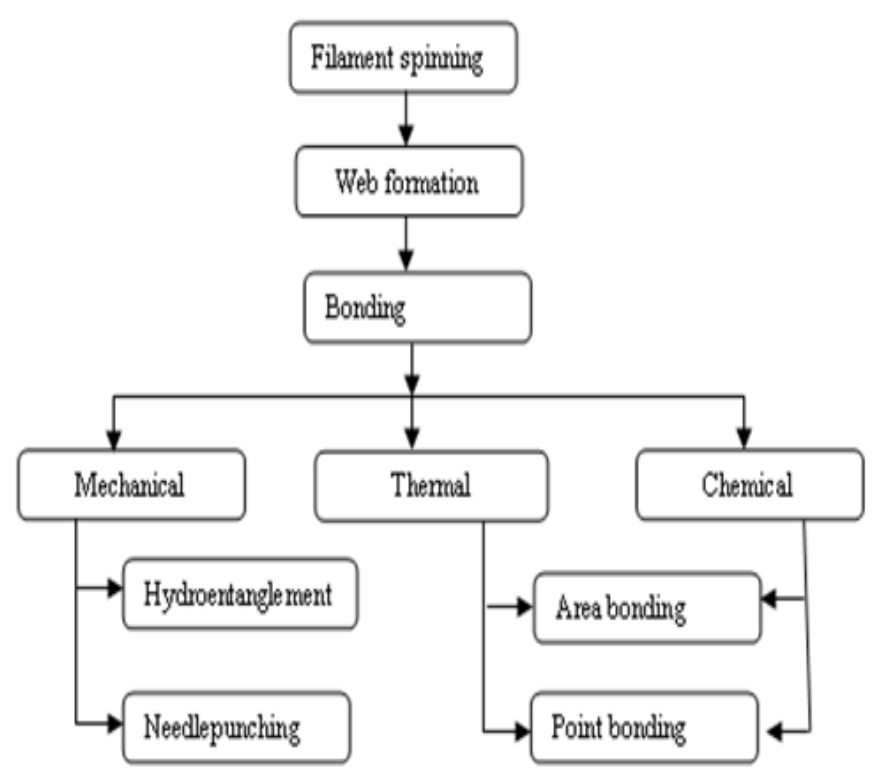

Figure I Steps in spun bonding process.

Filtration of the polymer melt is important for high quality filaments and fabrics besides a continuous and trouble free production. The line consists of two filters, one before the metering pump and other in the spinning block. ${ }^{7}$ In order to increase the life of the spin pack filter, 
the fineness of the metering pump filters is in the range of spin-pack filter or even lower. Selection of the filter must be such that it does not damage the melt or interrupt the melt flow. ${ }^{8}$ The polymer melt is then pumped into the spinning block by the metering pump. For bicomponent filaments, two independently driven metering pumps are used. The spinning block consists of spin block body, spin pack and spinneret. The spinneret is a single block of metal having thousands of drilled orifices on it. Several spinnerets are placed side by side to produce the wider webs. Design of spinneret influences the web quality. The orifice length depends on the pressure exerted by the metering pump but Olefins normally require a spinneret with longer length to diameter capillary.

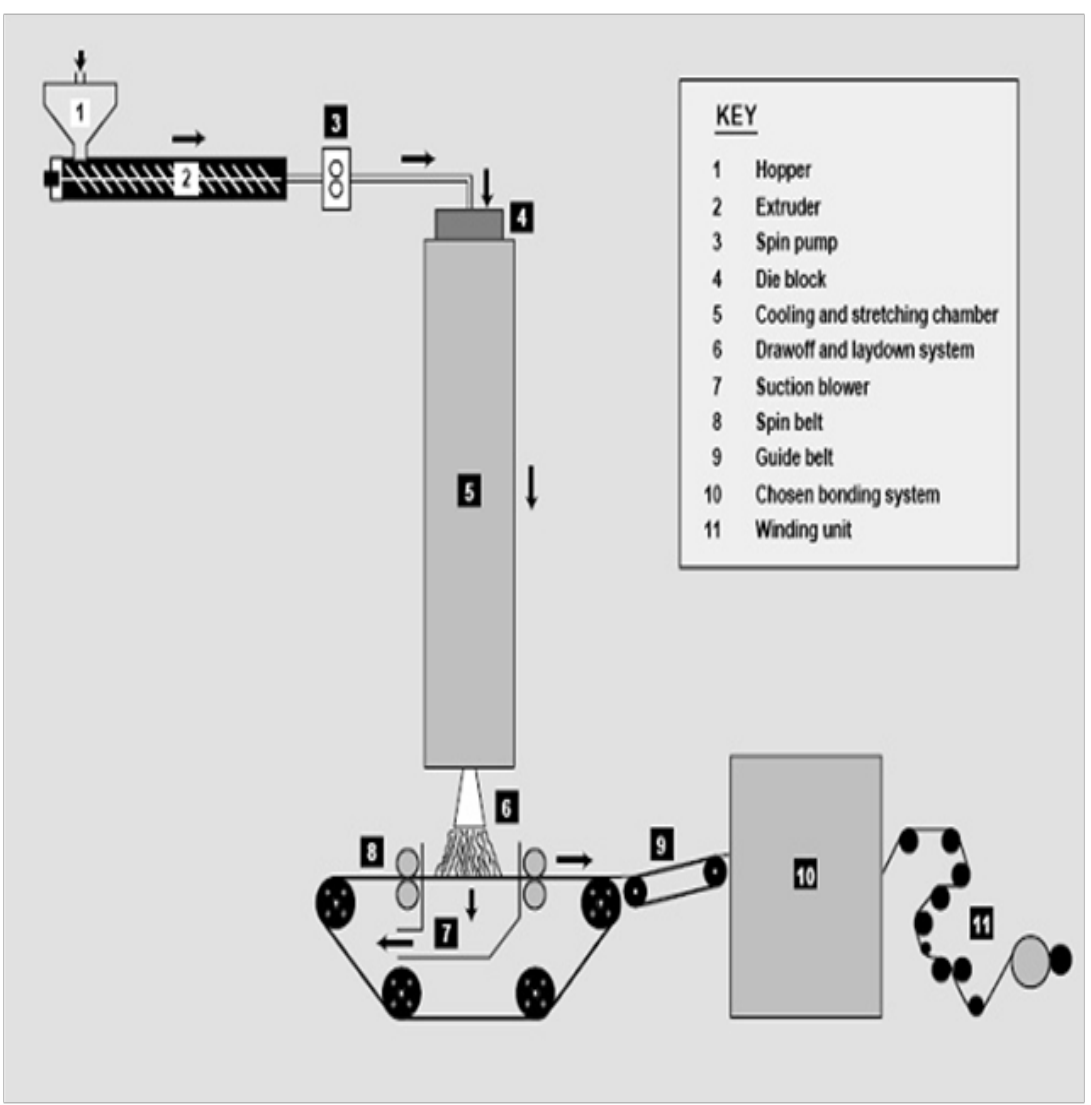

Figure 2 Schematic spun bonding process.

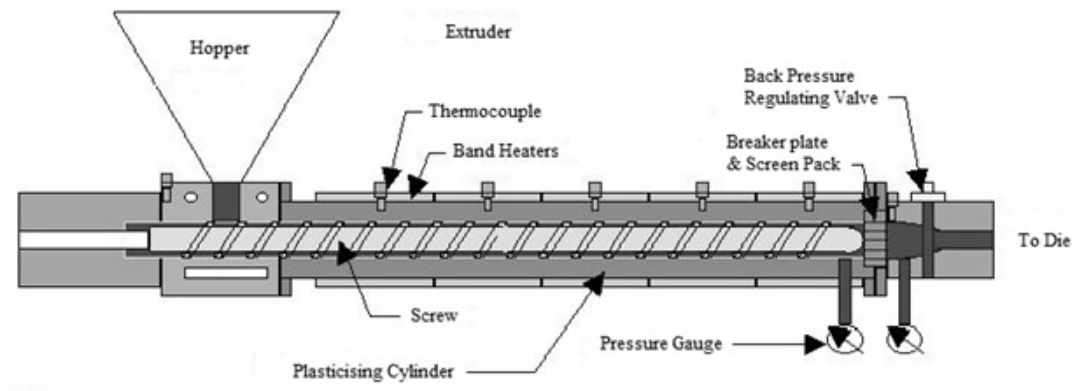

(A)

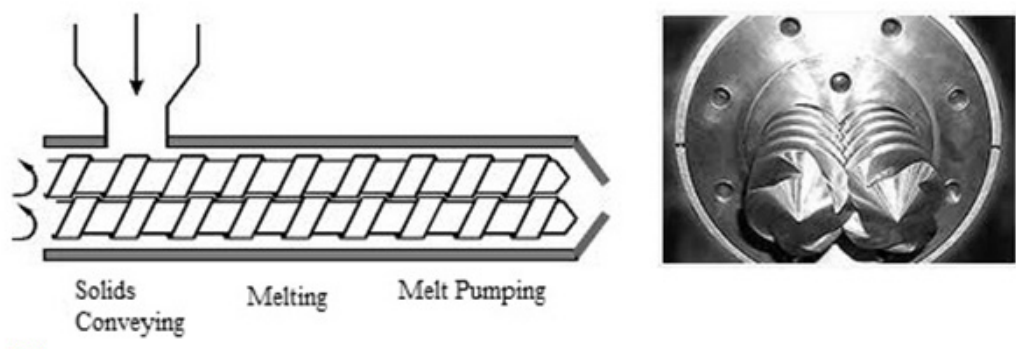

(B)

Figure 3 (A) Extruder; (B) Twin screw. 
The emitted filaments solidify on being subjected to cool air. Cross flow or inflow systems are used to supply conditioned air to the filaments. Simple quench air box, multi zone quench air box and quench air box for air conditioned zones are used as quench system. The quench air is generally introduced at a temperature that may vary from $5-55^{\circ} \mathrm{C}$ at a rate of $17-70 \mathrm{~m}^{3} / \mathrm{min} / \mathrm{m}$ of machine with nozzle opening from about $3-25 \mathrm{~mm} .{ }^{9}$ Filaments are provided strength by drawing. Attenuation of filaments leads to molecular orientation and improves the strength. Old spun bonding lines used godget rollers for drawing the filaments, but the production speeds were very low. ${ }^{10}$ In the new spun bonding lines the drawing takes place with the help of air (air speeds of 1.5 to 4 times the filament velocity) and it is possible to reach the filament speeds of $6000 \mathrm{~m} / \mathrm{min}$. depending upon the pressure of air, the technology is divided into three categories viz high pressure, low pressure and under pressure. ${ }^{11}$ Reifenhauser Reicofil spun bonding uses low pressure air system for processing polypropylene, polyethylene, polyester and polyamides. ${ }^{12}$ Polyester spun bond fabrics are difficult to be produced with negative attenuator pressure. Nordson's MicroFil TM spun bond system with a positive attenuator pressure makes it possible to produce polyester spun bond nonwovens with excellent properties. ${ }^{4,13,14}$ Bundle of filaments is deposited onto a moving belt randomly or in a required direction to form the web Figure 4. A suction box is located just below the forming belt. The individual filaments are separated mechanically, aerodynamically or electrically, before reaching the collecting belt in order to achieve maximum uniformity and cover. ${ }^{9}$ In electrical method of filament separation an electrostatic charge of the order of 30000 e.s.u. $/ \mathrm{m}^{2}$ is given to the filaments, which releases on contacting the grounded conductive belt. ${ }^{15}$ The filament separation in the spun bond fabric can be determined by calculating the bunching coefficient. ${ }^{16}$ It also helps to define the pore size distribution in the structure, which is important for applications like surgical gowns. ${ }^{17}$
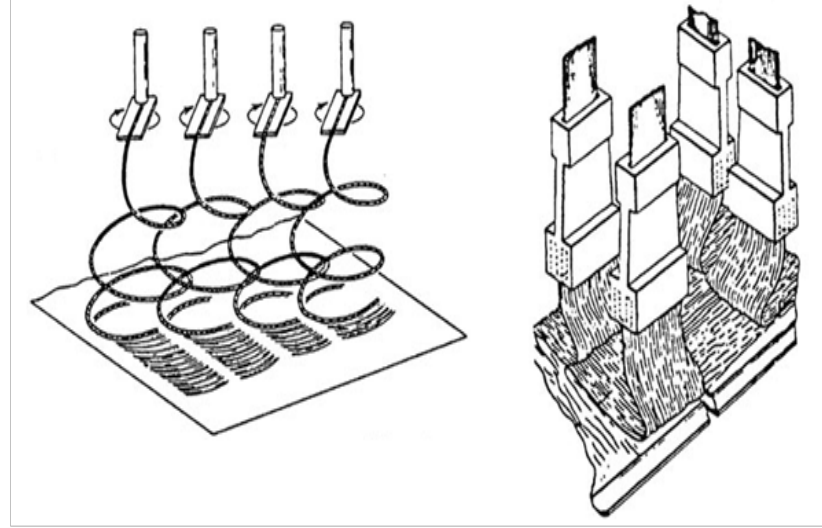

Figure 4 Filament lay down on the belt.

In order to achieve a particular characteristic in the final fabric, the directionality of the spun laid filament is controlled by traversing the filament bundles mechanically or aerodynamically as they move toward the collecting belt. In the aerodynamic method, alternating pulses of air are supplied on either side of the filaments as they emerge from the pneumatic jet. By proper arrangement of the spinneret blocks and the jets, lay down can be achieved predominantly in the desired direction. Highly ordered cross-lapped patterns can be generated by oscillating filament bundles. If the lay down belt is moving and filaments are being rapidly traversed across this direction of motion, the filaments are being deposited in a zigzag or sine wave pattern on the surface of the moving belt. The relationships between the collecting belt speed, the period of traverse, and the width of filament curtain being traversed determine the appearance of the formed web. ${ }^{4}$ Higher lay down belt speeds may lead to formation of blotches in the fabrics. ${ }^{17}$ When filaments are fed perpendicularly onto a moving belt, the lay-down form taken by the filament is determined by filament properties, such as linear density, bending rigidity, and torsional rigidity, the height of the feed point, and the feed-to-belt ratio. When a thread is being fed onto a moving belt, it will be laid in a modifiedcycloidal form, with a shape depending on the ratio between feed and belt speeds. With higher throughputs, the fiber diameters are larger and that leads to cycloids of larger diameter. ${ }^{1,5}$

Several bonding methods like thermal, chemical/adhesive, needling and hydro entanglement, available for staple fiber fabrics have been successfully adapted for continuous filaments. Higher production rates and flexibility have increased the use of needle punched fabrics particularly in geotextiles. Needle movement through the web at a rate of up to 2200 strokes/min can give as many as 500 penetrations $/ \mathrm{cm}^{2}$, depending on the needle density and line speed. The needle punched web tends to be more bulky than fabrics bonded by thermal or chemical binder methods. Since the fibers have freedom to move over each other, the fabric is easily deformed and exhibits low initial modulus. In addition, it is the only bonding method suitable for the production of heavyweight spun bond fabrics $\left(800 \mathrm{~g} / \mathrm{m}^{2}\right)$. Thermal and chemical bonding depends on fiber to fiber fusion for establishing fabric integrity. The degree of fusion viz. point or area bonding, determines many of the fabric properties like handle or softness. Thermal point bonding commonly used for light to medium weight webs results in the fusion of fibers at points, with fibers in between the point bonds remaining relatively free, leading to softer fabrics than area bonded. Point bonding is achieved in three steps, by subjecting the spun web into the nip of paired heated rolls, one of which has a raised pattern on its surface: ${ }^{18}$

\section{a. Heating the web to partially melt the crystalline regions \\ b. Diffusion of the newly released chain segments across the fiber to fiber interface \\ c. Subsequent cooling of the web to cause its re-solidification and to trap the diffused chain segments}

Bonding temperatures for polypropylene fibers usually do not exceed $170^{\circ} \mathrm{C}$, but pressures on the raised points are between 138 MPa-310 MPa. Typically, only $10-25 \%$ of the surface available for bonding is converted to fused, compacted areas of bonding.

For area thermal bonding the spun web is subjected to the source of heat, usually steam or hot air causing fusion of filaments at crossover points. The use of steam is limited to polypropylene and polyethylene fusion, since the pressures needed to reach the temperatures more than $200^{\circ} \mathrm{C}$ required for bonding polyesters are impractical. Area thermal bonding is based mainly on temperature and is applied to webs containing binder fibers. Complete fusion gives a paper-like structure with little resistance to tearing. The spun web may contain small amount of lower melting fiber, or the filaments may contain un-drawn segments which melt at lower temperatures than the fibers in the web. Hetero filament structures utilize a low melt sheath to effect fusion. Both polyethylene and nylon- 6 have been used as the low melting temperature sheaths in commercial spun bond products. Thermal bonding is far more common and economical than chemical bonding besides chemical bonding requiring thermal curing of the adhesives. ${ }^{4}$ Embossing, resin treatment, flame retardancy, rewet agent, 
dyeing, printing and antistatic finishes can be given to the bonded fabric depending upon the application. ${ }^{5}$

Further developments in spun bonding process have made it possible to produce micro fibers on spun laid machines giving the advantages of better filament distribution, smaller pores between the fibers for better filtration, softer feel and also possibility of making lighter weight fabrics. For this reasons spun laid production is increasing more rapidly than any other nonwoven process. ${ }^{4}$ Perfobond 3000 from Rieter is designed to lay down the filaments at $45^{\circ} \mathrm{C}$ from the machine direction, monitors and controls all critical process parameters every half meter crosswise making the fabric more uniform and even. ${ }^{19}$ Ason spun bonding technology incorporated the features of melt blowing process (ie, very high filament velocity, very high temperature of the filaments), adjustable collector to slot attenuator distance, in the spun bonding line and produce low bases weight fabrics with higher uniformity, which is comparable to melt blown fabric..$^{4,20}$ Moreover, it has reduced the building height required for the plant to only $6 \mathrm{~m}$ as compared to $10-25 \mathrm{~m}$ for low pressure to high pressure spun bond lines. A complex method of manufacturing spun bond fabrics known as 'Splash Spinning', involves spinning of heated pressurized solutions under precise conditions. Dupont uses this method to produce Tyvek Type 10 as area bonded and Type 14 and 16 as point bonded fabrics with unique structure and properties. A $10-$ $15 \%$ solution of high density polyethylene in trichloroflouromethane or methylene chloride is heated to $200^{\circ} \mathrm{C}$ and extruded through the spinneret at a pressure of $4.5 \mathrm{MPa}$ to get three dimensional film fibril network referred as plexi filaments. Multiple plexi filaments are thermally bonded to produce a sheet like fabric, which has high tenacity and soft handle. ${ }^{9}$

Reifenhauser's Reicofil employs two filament extrusion beams and two melt blowing sections, making it possible to produce various complex constructions like spun bond/melt blown/melt blown/spun bond (SMMS) for a variety of consumer and industrial applications. ${ }^{7,21}$ Netherlands based Akzo group is producing Colback (Colback type $\mathrm{A}$ and $\mathrm{N}$ in 80,100 and $120 \mathrm{~g} / \mathrm{m}^{2}$ for primary backing and Colback type NHD 30 and NHD 50 for secondary backing), a thermally bonded spun laid nonwoven produced from bicomponent filaments with polyester as core and polyamide as skin. Bicomponent lines require additional investment for second extruder, chip feed and more complicated and expensive spinning beam. Recycling the waste generated from bicomponent lines is difficult as compared to homo polymer sheets. ${ }^{22}$ Spun bonding has also been combined with hydro entanglement to combine the strength of filaments and drape of spun laced fabrics and it is possible to produce fabrics of weights under $10 \mathrm{~g} / \mathrm{m}^{2}$ to more than $600 \mathrm{~g} / \mathrm{m}^{2}$. Freudenberg Vliesstoffe has produced hydro entangled spun bonds named as Evolon made from 0.05-0.15 dtex filaments (polyester and polyamide), which is high tenacity isotropic fabric with high density and low air permeability. ${ }^{23-24}$

\section{Polymers used}

Although any polymer can be used in the spun bonding process, but polymers with high molecular weight and high molecular weight distribution like polypropylene, polyester, polyamides are commonly used for production of spun bond fabrics. High density polyethylene spun bond fabrics are also finding increasing applications. Isotactic polypropylene is the most widely used polymer for spun bond nonwoven fabrics. Because of its low density, it provides highest yield (fiber per kilogram) and covering power at the low cost.
Although unstabilized polypropylene is rapidly degraded by UV light, improved stabilizers permit several years of outdoor exposure before fiber properties deteriorate. Scrap or polypropylene fibers of inferior quality can be repelletized and then blended in small amounts with fresh polymer to produce first grade spun bond fabrics. ${ }^{1}$ Polypropylene fibres cannot be dyed easily since there are no dye receptor sites on the molecular backbone. Addition of a pigment to the polymer melt may be used to produce satisfactory color on the filaments.

Polyester (PET) is used in a number of commercial spun bond products and offers certain advantages over polypropylene, although it is more expensive. Unlike polypropylene, polyester scrap is not readily recycled in spun bond manufacturing. Tensile strength, modulus, and heat stability of polyester fabrics are superior to those of polypropylene fabrics. Polyester fabrics are easily dyed and printed with conventional equipment. But polyester fibres have lagged in their popularity due to economics and processing concerns. Polyester fibres are extruded at relatively higher temperature and the resin should be dry before melting and extrusion. It is easier to spin PP fibres with finer diameter than PET. ${ }^{9,13}$

Spun bond fabrics made from nylon- 6 and nylon-6,6 are highly energy intensive and expensive than polyester or polypropylene. Nylon-6,6 spun bond fabrics are produced with weights as low as $10 \mathrm{~g} / \mathrm{m}^{2}$, offer ease of bonding, lower rigidity, Dyeability, excellent cover, strength, extensibility and resilience. ${ }^{9}$ The properties of melt spun polyethylene fibers by traditional methods are inferior to those of polypropylene fibers.

Polyurethanes and many types of rayons have been successfully processed into usable spun bond webs. The main advantage of rayon is that it provides good drape and softness to web. Improved bonding and higher fabric strength could be achieved through the use of bicomponent fibers, such as sheath-core or islands-in-the-sea (I/S) fibers, in which numerous small filaments of one polymer (islands) are placed in a matrix of another polymer (sea). However, the island (core) and sea (sheath) components must have certain characteristics.

i. The island polymer should have higher strength and lower elongation at break than the sea component.

ii. The sea should have a lower melting temperature than the island.

Moreover, only in the presence of a strong interface between the island and sea polymers, the mechanical stresses could be transferred between weak sea and strong islands. If the sea and island materials comply with all previously mentioned requirements, then it is probable that the thermal bonding process would not influence the morphology and the strength of the islands in the vicinity of the bond spots, whereas the sea could be completely melted acting as a binder and transferring the stress to stronger island fibers under the load. ${ }^{18}$ In the past few years there has been a growing interest and increased production of bicomponent spun bond nonwovens, particularly with a polypropylene core and polyethylene sheath. DSC and TMA scan of PP homo polymer and PP/PE copolymer filaments shows that PP homo polymer has better thermo mechanical properties than the PP/ PE copolymer filaments because the PP homo polymer has higher crystallinity and molecular orientation than the PP/PE copolymer. Therefore, PP homo polymer has higher tensile strength and initial modulus and lower breaking elongation than the copolymer. The peak melting temperature and enthalpy of melting for co-polymer 
are lower than those of the homo polymer. Therefore the bonding and processing temperatures of the co-polymer are lower than that of homo polymer. ${ }^{25-27}$ Use of copolymer (with ethylene contents of $3-5 \%$ ) relative to polypropylene shifts the brittle point (Tg) to a lower temperature, giving higher impact strength at a low temperatures and therefore lower fabric stiffness, ${ }^{26}$ High strength micro and nano fiber based spun bond nonwovens have been produced by spinning 'Islands in Sea' fibres made up of Nylon 6 as islands and Polylactic acid (PLA) as sea. ${ }^{28}$

\section{Fabric characteristics and properties}

The structure of woven and knitted fabrics permits the fibres to move readily within the fabric when in-plane shear forces are applied, resulting in better conformability, whereas calendar bonding of a spun web causes fibres to fuse and impart integrity to the sheet. The resulting structure has a stiffer handle or drape due to immobilization of the fibres in the area of fusion. The effect can be moderated by limiting the bonds to very small areas (points) or by entangling the fibres mechanically (needle punching or hydro entanglement). Saturation bonding of spun webs with chemical binders like acrylic emulsions can bond the structure throughout to give stiff sheets.

Spun bond webs offer product characteristics ranging from very lightweight and flexible structures to heavy and stiff structures, with property combinations falling between paper and woven fabrics. The main characteristics and properties are: ${ }^{2}$

A. Random fibrous structure

B. Generally white with high opacity

C. High strength-to-weight ratios compared to other nonwoven, woven, and knitted structures

D. High tear strength

E. Planar isotropic properties due to random lay down of fibers. However optical analysis of the fibrous orientation distribution shows that the conveyor face of the fabric, which is in contact with the conveyor belt is more anisotropic than the upper facz. ${ }^{19}$

F. Good fray and crease resistance

G. High liquid retention capacity due to high void content

H. High in-plane shear resistance

I. Low drape ability

J. Most are layered or shingled in structure; the number of layers increase as basis weight increases

$\mathrm{K}$. Basis weight ranges from 5 to $800 \mathrm{~g} / \mathrm{m}^{2}$ but is typically $10-200 \mathrm{~g} /$ $\mathrm{m}^{2}$.

L. Web thickness ranges from 0.1 to $4.0 \mathrm{~mm}$ but is typically $0.2-$ $1.5 \mathrm{~mm}$.

M. Fiber diameter ranges from 1 to $50 \mu \mathrm{m}$, but the preferred range is $15-35 \mu \mathrm{m}$.

Random fibre structure, breathability, resistance to fluid penetration, lint free structure, sterilizability and impermeability to bacteria make the fabrics suitable for use in medical field like disposable operating room gowns, shoe covers and sterilizable packaging. ${ }^{12}$

\section{Process variables}

Fabric properties are affected by the properties of the filaments and other process parameters like air speed in distribution chamber, belt speed, through put rate, calendaring time, calendar surface temperature and nip pressure. ${ }^{15}$ The structure and properties of the filaments are determined by the dynamics of the thread line and the effect of air drag on spin line, which are dependent on elongation deformation and crystallization during solidification. ${ }^{1}$ The orientation distribution of filaments can be adjusted by changing speed of conveyor belt and aspirator. The orientation in cross machine direction increases as the oscillating velocity of the aspirator increases. Increasing the conveyor belt velocity increases the orientation in machine direction. ${ }^{29}$ Fibre density, temperature resistance, chemical and light stability and ease of coloration depend on the base polymer. ${ }^{2-3}$

The major process variables that affect the properties of spun bond fabrics are: ${ }^{16}$

I. Filament variables:
a. Linear density
b. Tenacity
c. Elongation
d. Modulus
e. Cross section
f. Crimp
g. Morphology

Filament arrangement:

i. Filament separation

ii. Fabric weight uniformity

iii. Filament orientation

II. Bonding variables:
a. Binder nature
b. Binder concentration
c. Binder distribution
d. Self bonding

The effect of various process parameters on the filament properties and fabric properties is discussed in the following sections.

\section{Polymer melt temperature}

The filament diameter decreases as melt temperature increases, because lower viscosity at higher melt temperature allows easier draw down of filaments. Draw down and diameter reduction is difficult at lower temperatures but possibility of polymer degradation, leading to filament breaks and spot formation on spin belts exists at higher temperatures. Therefore careful selection of the melt temperature is important. Lower diameter filaments show higher birefringence values indicating higher orientation and crystallinity, leading to higher tensile strength and lower breaking elongation. ${ }^{1,30,31}$ 


\section{Throughput rate}

Filament diameter increases as throughput rate increases, in spite of an increase in air suction to keep a balance of air-to-polymer ratio. Higher throughput makes it difficult to drawdown the filaments resulting in poor crystallinity of the filaments and the spun bond fabric. The birefringence of filaments also decreases as the throughput rate increases. TMA scan of the filaments shows that for the same primary air temperature the filaments become more stable with decreasing throughput. The morphology of the filaments spun at lower throughput is better developed than those with higher throughput because rheological conditions are more favorable for crystallization and orientation at lower throughputs. ${ }^{1,26,27}$

Tensile strength and burst strength of spun bond fabrics decreases and elongation at break increases as throughput rate increases. Fabric tear strength decreases with increased throughput rate at the beginning and then increases as throughput increases further because tear strength is influenced by filament strength and fabric density. Air permeability increases as the throughput rate increases due to increased filament diameter. Increase in throughput requires increased bond temperature to attain optimum transfer of heat and to produce a fabric with optimum properties. ${ }^{32}$

\section{Primary air temperature}

Fibre diameter decreases with increase in primary air temperature. Increased quench air temperature decreases the fibre diameter owing to lower viscosity in the fibre formation zone, thus allowing higher draw ability. Increased quench air temperature also decreases the thread line stress, delays the crystallinity and reduces effective drawdown. This effect is more prominent in the absence of positive take-up mechanism in the spun bonding process. Therefore, fibre diameter may increase with increase in quench air temperature. Fine filaments have better morphology and properties compared to large diameter filaments.

The tensile strength of filaments decreases and elongation at break increases with increased cooling air temperature due to higher orientation of molecules in filaments produced at low primary air temperature than those produced at high primary air temperature. ${ }^{26}$ The TMA scan of filaments shows that filaments produced at lower primary air temperatures are more stable in their thermo mechanical response. Similar thermo mehanical behavior is observed in the produced nonwoven fabrics.

Crystallinity of nonwoven fabrics was found to decrease as the primary air temperature increases, which is similar to that observed in the filaments. Tensile strength, tear strength and burst strength of the bonded nonwoven fabric decreases and elongation at break increases, as primary air temperature increases. Increased filament diameter at higher primary air temperature leads to lower fabric density and higher air permeability of bonded fabrics.

\section{Molecular weight}

Increasing molecular weight with same molecular weight distribution leads to higher spin line stress resulting in faster filament draw down, higher crystallization rates, higher levels of crystallinity and orientation, higher tensile strength and lower breaking elongation for filaments. Nadella et al. reported that crystalline orientation in melt spun fibres is determined by spin line stress rather than take up velocity and draw down. ${ }^{33}$ Broad molecular weight distribution polymers show more elongation, thinning and higher tendency to undergo stress-induced crystallization, resulting in fibers with higher density and lower birefringence than the filaments produced from narrow molecular weight distribution. Fibers spun from narrow molecular weight distribution have higher tensile strength and lower elongation to break. ${ }^{34}$

\section{Quenching air pressure}

An increase in quenching air pressure, which is accomplished by adding more auxiliary air, resulted in a decrease in the fiber diameter. An increase in air pressure leads to an increase in the spin line draw ratio of the filaments.

\section{Air suction}

Air suction has an obvious effect on the fiber structure as the air suction directly corresponds to take-up speed. The fiber diameter decreases with an increase in the air suction speed.

\section{Polymer type}

A comparison of the tensile properties of metallocene PP and zigler nata PP shows that $\mathrm{mPP}$ filaments had much higher strength and lower breaking elongation although $\mathrm{mPP}$ filaments have less developed morphology reflected in lower crystallinity values. With $\mathrm{mPP}$, higher strength is realized at a relatively lower bonding temperature because of better melting of mPP fibres and formation of good bonds due to sufficient polymer flow. A comparison of the tensile properties of spun bond fabrics made from metallocene PP and zigler nata PP filaments shows that the fabric strength increases with increase in the bonding temperature. However, the strength drops off after reaching a maximum bonding temperature, which is $140^{\circ} \mathrm{C}$ for $\mathrm{znPP}$. $\mathrm{mPP}$ has a wider bonding temperature range as compared to $\mathrm{znPP} .^{30,32}$

Metallocene based isotactic propylene polymers have been used in spun bonding process and commercialized in early 1995. The metallocene based catalyst offer higher versatility and flexibility for both the synthesis and the control of structures of polyolefins over the conventional ziegler natta catalyst system due to their identical reactive sites. The advantages of metallocene catalyst are:

a. High strength to weight ratio

b. narrow molecular weight distribution

c. lower melting point

d. Cost-effectiveness.

e. Metallocene resins are stiffer than the conventional resins.

\section{Bonding technique}

Thermal point bonding, most commonly used in spun bond fabrics affects the internal fibre structure, causing an important drop in fabric tensile properties. A reduction in birefringence (molecular orientation) of the fibres has been observed at the bond spot and in its vicinity for isotactic polypropylene (iPP) and polyethylene terephthalate (PET) nonwovens. The molecular orientation of the fibers entering the bond spot decreased significantly over a distance of 30-40 microns from the bond edge. The change in the fiber morphology was influenced by bonding conditions and it was significantly smaller in samples bonded at lower temperatures or for shorter times. The birefringence of the fibers is low within the bond and at its edge, but the cross-sectional area of the bond is much larger than that of the fibers, thus the fibers at the bond periphery fail predominantly. Because the change in the fiber morphology is influenced by bonding conditions, over-bonded 
webs demonstrate a larger loss of mechanical properties of the fibers entering the bond compared to well-bonded webs.

In adhesive bonding process, the adhesive squeezed through the perforations of screen deposits on the fibre web keeping the fibre layout unchanged. Moreover, adhesives being the surface agents do not affect the internal structure resulting in better tensile properties. Flexible adhesives (UV 422 and 4693-H) give better results than structural adhesives (Olé Bond 2) in terms of fabric tensile properties even though the interfacial bonds were weak. ${ }^{35}$

Kothari and Das studied the compression behavior of heat sealed and needle punched spun bond fabrics. ${ }^{36}$ It was observed that the bulk density of heat sealed spun bond fabrics is much higher than the needle punched spun laid fabrics. In the needle punched fabrics, the fibres are held together in their respective positions only by frictional contacts but in heat sealed fabrics, the adjacent fibres are fused together. Therefore, heat sealed fabrics show lower compressibility and better resilience as compared to needle punched fabrics. In heat sealed spun bond fabrics, there is little chance of filament to filament slippage during compression, but in needle punched spun bond nonwovens, the chance of filament to filament slippage is higher.

Measurement of filament diameter before and after bonding shows that the diameter of the filaments after bonding is $10-13 \%$ more than that before bonding.

\section{Bonding temperature}

Tear and abrasion properties needed in applications like crop covers are controlled by the amount of thermal calender bonding. ${ }^{37}$ Tear strength increases and abrasion resistance decreases as the amount of thermal calender bonding is decreased (by decreasing calender temperature and pressure). In a study on the cotton surfaced polypropylene spun bond fabrics, it was observed that bursting strength, flexural rigidity, specific stress and breaking elongation increase and air permeability decreases as the bonding temperature increases. $^{38}$

Nanjundappa and Bhatt analyzed the nature of bonding between filaments to understand the mechanism of rupture of polypropylene spun bond fabrics. ${ }^{38}$ It was observed that the throughput rate and bonding temperature influence the filament properties and determine the nature of bonds. Increasing bonding temperature and/or decreasing throughput rate causes the filaments to flatten and offer more surface area during bonding, making it more coherent. In case of samples bonded at low bonding temperature or high throughput rate, the rupture proceeds with disintegration of the bond area in comparison to the samples bonded at high bonding temperature or low throughput rate. Filaments peel off from the bond area as the stress builds up during tensile loading, until the bond disappears. These samples exhibit low peak stress and high breaking strains. Final rupture occurs with the breaking of these filaments. In case of samples bonded at higher bonding temperature or low throughput rate, the bond area is strong because of larger bonding areas of filaments. Moreover the bond peripheries are weak because higher bonding temperature or contact time makes the polymer squeeze out of the bond and solidify under no tension producing a brittle structure. ${ }^{39}$ In such cases, the bond periphery ruptures first. Under optimum conditions, the rupture occurs due to filament separation from the bond areas, but the bond itself is deformed and bond disintegration occurs at higher stress levels than those bonded at low temperatures. Some points of rupture also occur at the bond periphery. Because of combination of stronger bonding and extension of bonds, the sample exhibits highest breaking load.

\section{Conclusion}

Nonwoven fabrics made by spun bond process are being used in various applications like disposable diapers, hygiene and healthcare textiles, filtration, automotive industry, civil engineering, packaging, carpet backings. The consumption of spun bond fabrics in durable and disposable products is continuously growing and the development of new products with hydro entanglement of spun bonds is expected to further increase the market share of spun bond fabrics.

\section{Acknowledgments}

None.

\section{Conflict of interest}

Author declares there is no conflict of interest in publishing the article.

\section{References}

1. Bhat GS, Malkan SR. Extruded continuous filament nonwovens: Advances in scientific aspects. J Applied Poly Sci. 2002;83(3):572-585.

2. Malkan SR. An overview of spun bonding and melt blowing technologies. Taapi J. 1995;78:185-190.

3. Bhat GS, Kotra R. Development of structure and properties during spun bonding of metallocene catalysed popropylene. Polymer-Plastic Technol and Eng. 2008;47(5):542-549.

4. Midha VK, Kothari VK. Developments and recent trends in spun bonding. The Indian Tex J. 2004;114:17-24.

5. Lim H. A review of spun bond process. J Tex \& Apparel, Technol \& Manage. 2010;6(3):1-13.

6. Tokunaga A. Uptodate spun bond technology. International Textile Bulletin-Nonwoven/Industrial Textiles. 1998;44:21-24.

7. Schmidt O. Polymer filtration in the spun bond production. Technical Textiles. 2001;44:E159-E160.

8. James A. Producing nonwoven fabrics by melt extrusion: An introduction to melt blown and spun bond processes. International Fiber J. 2000. p. 32-35.

9. Mark HF, Bikales NM, Overberger CG, et al. Encyclopedia of Polymer Science and Engineering. New York: Wiley-Interscience; p. 227-253.

10. Hajji N, Spruiell JE, Lu FM, et al. INDA Journal of Nonwoven Research. 1992;4:16.

11. Wilson A. Polymer technology and rapid change will characterize the industry. Nonwoven Report International. 1998;325:32-37.

12. Kunze B. Nonwoven production with spun laid technology. ITB Nonwovens-Industrial Textiles. 1998;3:41-45.

13. Wang X, Michielsen S. Morophology gradients in thermally point bonded poly (ethylene terephthalate) nonwovens. Text Res J. 2002;72(5):394-398.

14. Wang $\mathrm{H}$, Jin $\mathrm{X}, \mathrm{Wu} \mathrm{H}$. The influence of slot width of an attenuator on the properties of PET spun bonded fibers. J Material Sci. 2007;42(19):8006-8009.

15. Goswami BC. Spun bonding and melt blown processes. Manufactured Fibre Technology, London: Chapman \& Hall. 1997. p. 561-594. 
16. Ericson CW, Baxter JF. Spun bonded nonwoven fabric studies-I: Characterization of filament arrangement in the web. Text Res J. 1973;43(7):371-378.

17. Shealy OL. Spun bond products- A new concept in utilization of fibrous materials. Text Res J. 1965;35(4):322-329.

18. Fedorova N, Verenich S, Pourdeyhimi B. Strength optimization of thermally bonded spun bonded nonwovens, J Eng Fibers \& Fabrics. 2007;2(1):38-48.

19. Salvado R, Silvy J, Drean J. Relationship between Fibrous Structure and Spun bond Process. Text Res J. 2006;76(11):805-812.

20. Fu ML. Ason achieves breakthrough in spun bonded process. International Fiber J. 1997. p. 49-50.

21. Wadsworth L, Zhang D, Sun Q, et al. The spun bond and melt blown story. Nonwoven Industry. 1992. p. 62-74.

22. Fischer G. Technological knowhow per square centimeter. Textile Horizons. 1990. p. 37.

23. Watzl A. Spun bonding and spun lacing. Technical Textiles. 2001;44:E167-E172.

24. Schuster M. Evolon-A new generation of technical textiles. Technical Textiles. 2001;44:2.

25. Wadsworth L, Sun Q, Zhang D, et al. SM and SMS Laminates Produced with $100 \%$ PP Melt blown and Bicomponent Fiber PP/PE Melt blown Webs. Nonwoven Industry. 1999;30:68-74.

26. Zhang D, Bhat G, Malkan GS, et al. Evolution of Structure and Properties in a Spun bonding Process, Text Res J. 1998;68(1):27-35.

27. Zhang D, Sun Q, Bhat G, et al. Structure and property characterization of spun bonded filaments and webs using thermal analysis. J Applied Polymer Sci. 1998;69(3):421-434.

28. Fedorova N, Pourdeyhimi B. High strength nylon micro and nano fiber based nonwovens via spun bonding. J Applied Polymer Sci. 2007; 104:3434-3442.
29. Jeon BS. Theoretical orientation density function of spun bonded nonwoven fabric. Text Res J. 2001;71(6):509-513.

30. Bhat GS, Nanjundappa R, Kotra R. Development of structure and properties during spun bonding of propylene polymers. Thermochimica Acta. 2002;392-393:323-328.

31. Zhang D, Bhat G, Malkan S, et al. Development of the structure and properties of polypropylene copolymer and homo polymer filaments during a spun bonding process. $J$ the Text Inst. 1998;89(2):289-303.

32. Nanjundappa R, Bhat GS. Effect of processing conditions on the structure and properties of polypropylene spun bond fabrics, J Applied Polymer Sci. 2005;98(6):2355-2364.

33. Nadella HP, Henson HM, Spruiell JE, et al. Melt Spinning of Isotactic Poly proylene: Structure, Development and Relationship to Mechanical Properties. J Applied Polymer Sci. 1977;21(11):3003-3022.

34. Misra S, Lu FM, Spruiell JE, et al. Influence of molecular weight distribution on the structure and properties of melt spun polypropylene filaments. J Applied Polymer Sci. 1995;56:1761-1779.

35. Limem S, Warner SB. Adhesive point bonded spun bond fabrics. Text Res J. 2005;75(1):63-72.

36. Kothari VK, Das A. The compressional behavior of spun bonded nonwoven fabrics. $J$ the Text Inst. 1993;84(1):16-30.

37. Avril D. An Innovative Approach to Spun bond Agricultrual Crop Cover. $J$ the Text Inst. 2001;30(4):311-319.

38. Sun CQ, Zhang D, Wadsworth L, et al. Processing and Property Study of Cotton-Surfaced Nonwovens. Text Res J. 2000;70(5):449-453.

39. Warner SB. Thermal bonding of polypropylene fibers. Text Res J. 1989;59(3):151-159. 